\title{
A PURELY ALgEBRAIC PROOF OF AG INEQUALITY
}

\author{
IVICA GUSIĆ
}

Abstract. We give a purely algebraic proof of AG inequality. We also give some examples.

Mathematics subject classification (2000): 26D05, 11P81, $05 \mathrm{E} 05$.

Key words and phrases: AG inequality, partition, quasi-sum of squares.

\section{REFERENCES}

[1] P. S. Bullen, Handbook of Means and Their Inequalities, Kluwer Academic Publishers, Dordrecht/Boston/London, 2003.

[2] I. GUSIĆ, A noncommutative AG inequality (preprint)

[3] A. HuRWITZ, Über den Vergleich des arithmetischen und des geometrischen Mittels, J.Reine Angew. Math., 108, (1891), 266-268.

[4] I. G. Macdonald, Symmetric Functions and Hall Polynomials, Clarendon Press, Oxford, 1979. 